\title{
LA ECOLOGIA POLITICA MARXISTA Y DECOLONIAL, EN EL LAGO DE TOTA BOYACÁ, COLOMBIA
}

\author{
Laura Angélica Puentes Puentes ${ }^{1 *}$
}

1 Universidade Federal da Integração Latino-Americana. Foz do Iguaçu, 85866-000, Brazil.

$$
\text { *laanpupu@gmail.com }
$$

Submitted: 16/09/2019. Accepted: 23/11/2019

Published: 26/11/2019

\section{RESUMEN}

Propósito: El objetivo es observar las complejidades en las condiciones socioambientales y en la justicia ambiental del lago de Tota, Boyacá, Colombia.

Metodología / Foco: La metodología a ser utilizada es la pesquisa cualitativa, por medio del método de estudio de caso, pues permite identificar el comportamiento de los individuos frente a un fenómeno, en un contexto social.

Resultados: De acuerdo con lo expuesto, este importante ecosistema es un escenario para reflexionar sobre la persistencia del olvido por parte de la entidades gubernamentales y habitantes locales, la actitud de ellos es preservar el lago para producir riqueza, explotar la tierra y los recursos naturales.

Limitación / desenvolvimiento de la investigación: El contenido de este análisis no es suficiente para entender la compleja situación social y ambiental, entre la población y el lago.

Originalidad / Valor del artículo: Este articulo presenta algunas definiciones que ayudan a entender un poco más la Ecología Política, en cuyos puntos de basa este análisis: injusticia y racismo ambiental, conflictos y problemas ambientales, desarrollo segregacionista, zona de sacrificio, inseguridad y dependencia alimentaria, movimientos sociales, modernidad/ colonialidad, la identidad y el papel de la mujer, permitiendo entender la complejidad de la situación actual de ese ecosistema.

PALABRAS CLAVES: ecología política, recurso hídrico, lago de Tota, soberanía alimentaria.

\section{MARXIST AND DECOLONIAL POLITICAL ECOLOGY, IN THE LAKE OF TOTA BOYACÁ, COLOMBIA}

\section{ABSTRACT}

Purpose: The objective is to observe the complexities in social and environmental conditions and environmental justice in Lago de Tota, Boyacá, Colombia.

Methodology/Approach: The methodology to be used is the qualitative research, through the case study method, as it allows to identify the behavior of individuals facing a phenomenon, in a social contexto.

Findings: According to the above, this important ecosystem site is a scenario to reflect on the persistence of oblivion by government entities and locals, their attitude is to preserve the lake is to produce wealth, exploit land and natural resources.

Research Limitation/implication: The content of this analysis is not sufficient to understand the complex situation social and enviromental, between the population and the lake.

Originality/Value of paper: This article presents some definitions that help to understand Political Politics a little more, whose items are based on the analysis: environmental injustice and racism, environmental conflicts and problems, segregationist development, zone of sacrifice, food insecurity and dependence, social movements, modernity / coloniality, identity and the role of women, which allows us to understand the complexity of the current situation of this ecosystem.

KEYWORDS: political ecology, hidric resource, Tota lago, food sovereignty. 


\section{INTRODUCCIÓN}

Por medio de este trabajo se busca generar un estudio de caso, desde la Ecología Política Marxista y Decolonial, reflexionando sobre ítems puntuales, como la injusticia y racismo ambiental (Pacheco, 2007), los conflictos y problemas ambientales, el desarrollo segregacionista, considerando este lugar como zona de sacrificio, la inseguridad y dependencia alimentaria, los movimientos sociales, la modernidad/ colonialidad, la identidad y el papel de la mujer. Estos puntos permitirán entender las trasformaciones y el futuro del lago; lo que representa para la población y reconocer las acciones de los agentes sociales en este lugar. Por otro lado, cabe resaltar que el contenido de este análisis no es suficiente para entender la compleja situación social y ambiental, entre la población y el lago.

Según el Estudio Nacional del agua 2014, "el Lago de Tota es el tercero más grande de Latinoamérica después del Maracaibo y del Titicaca, con un área de casi 5.600 hectáreas" (Instituto de Hidrología, Meteorología y Estudios Ambientales. 2014, p. 88). En el Lago de Tota, existe población campesina que se abastece del agua para consumo humano, aproximadamente 250.000 (Departamento Nacional de Planeación, 2014, p. 10) habitantes del Departamento de Boyacá y para el riego de cultivos de cebolla, papa, haba y arveja. Se usa para el criadero de trucha, 652 toneladas (Hidrosfera, 2012). También para atender a los turistas que recibe el sector hotelero. Así mismo es significativo este lugar por producir, 131.451 toneladas de cebolla por año (Departamento Administrativo Nacional de Estadística, 2001, p. 17), que abastece grandes ciudades como Bogotá, Tunja y Sogamoso. De acuerdo a lo anterior y retomando a Hardin quien expresa que: "La ruina es el destino hacia el cual corren todos los hombres, cada uno buscando su mejor provecho en un mundo que cree en la libertad de los recursos comunes" (Hardin, 1968, p. 1244), se observa la extracción de bienes y servicios para determinada población, donde cabe preguntarnos ¿Quiénes son los que realmente se perjudican y que realmente están haciendo los diversos agentes sociales, políticos, económicos e industriales por conservar el Lago?

El presente trabajo se compone, en una primera parte de la introducción; luego se encuentra la base teórica en la cual se fundamentó el desarrollo del tema, definiendo la Ecología Política y los puntos a analizar en este estudio de caso; una vez claros estos conceptos se realizan los resultados y discusión específicamente para el lago de Tota y por último se concluye con las consideraciones finales.

\section{FUNDAMENTACIÓN TEORICA Y CONCEPTUAL}

Partiendo de la base teórica propuesta por Blaikie y Brookfield "La ecología política combina las inquietudes de la ecología (antropología ecológica), y una economía política ampliamente definida. Juntos abarcan la dialéctica constante del cambio entre la sociedad y los recursos naturales, y también entre las clases y los grupos dentro de la sociedad misma" (BLAIKIE y BROOKFIELD, 1987; CALDERÓN-CONTRERAS, 2013). Para entender un poco más la Ecología Política Marxista y Decolonial, retomaremos a varios autores, entre los cuales están Joan Martínez que define la ecología política como el "estudio de los conflictos de distribución ecológica, conflictos en el acceso y en el control sobre los recursos naturales, particularmente como una fuente de subsistencia, así como los costos por la destrucción ambiental" (Martínez, 2004, p. 21). Para Agustin Lao-Montes es "redefinir las relaciones sociales entre los seres humanos, y de los humanos con otras formas de vida y con el resto del cosmos, de relaciones de apropiación, competencia y explotación, a relaciones de armonía, reciprocidad $y$ complementariedad, son algunas de las premisas principales de la política ecológica" (LaoMontes, 2011, p.161). También Escobar afirma que "es por medio de la cual se buscan definir los actuales problemas socioambientales" (Escobar, 2010, p.11), se parte de la interpretación que se le 
da al territorio, al capital, a la naturaleza, al desarrollo, a la identidad y las redes. Esta herramienta teórico analítica busca entender la relación dicotómica entre hombre y ambiente. Por su parte Leff la define como la construcción de un futuro sustentable, de un nuevo territorio del pensamiento crítico y de la acción política para "explorar con nueva luz las relaciones de poder que se entretejen entre los mundos de vida de las personas y el mundo globalizado" (Leff, 2003, p. 22). Es necesario adoptar no solo una perspectiva constructivista sino también una perspectiva política (STRACCIA, 2019).

La Ecología Política Marxista y Decolonial permite reflexionar como lo menciona Bookchin "como una sociedad verdaderamente libre, basada en principios ecológicos, podría mediar en la relación humana con la naturaleza" (Bookchin, 1999, p. 17). La ecología política, surge en la década de los años setenta, como resultado de la unión entre la ecológica y la economía política. Hoy en día, es un campo interdisciplinario que se complementa con otras ciencias. Así mismo recurre a la teoría liberal, al marxismo, postestructuralismo, a la teoría feminista, a la fenomenología, a la teoría postcolonial, y se acerca a la ciencia natural como ecología del paisaje y biología de la conservación.

Teniendo en cuenta lo anterior, a continuación se deducen y definen los aspectos que abarcan la ecología política decolonial y marxista:

Injustica y racismo ambiental: este concepto nace "a partir de la movilización contra un depósito de residuos tóxicos en el Condado de Warren, Carolina del Norte, entre 1978 y 1982, se descubrió que tres cuartos de ese tipo de entierros, estaban localizados en barrios habitados por negros" (Pachecho, 2007), también es la reclamación para recuperar la cultura y tradiciones originarias de poblaciones marginalizadas, que se les ha llevado al paradigma del enblaquecimiento.

Conflictos y Problemáticas Ambientales: es el daño que se ocasiona a los recursos naturales, generalmente viene de actores exógenos, sin embargo no se puede considerar el conflicto ambiental sin dimensión social (Fontaine, 2004; Massa-Sánchez et al., 2018). Hardin explica en la Tragedia de los comunes, "aquí el asunto no es sacar algo de los recursos comunes, sino de ponerles algo dentro -drenajes o desechos químicos, radioactivos o térmicos en el agua; gases nocivos o peligrosos en el aire; anuncios y señales perturbadoras y desagradables en el panorama" (Hardin, 1968).

Desarrollo segregacionista: la palabra desarrollo se define como avance, progreso que ha sido heredado de occidente, Valcárcel retoma a Robert Nisbet quien concibe que "la idea de progreso sostiene que la humanidad ha avanzado en el pasado - a partir de una situación inicial de primitivismo, barbarie o incluso nulidad- y que sigue y seguirá avanzando en el futuro" (Valcárcel, 2006, p. 5), y la palabra segregacionista, es usada por los europeos para excluir, para apartar. Así, el desarrollo segregacionista es apartar a determinada población, comunidad para alcanzar un llamado progreso.

Zona de sacrificio: el término se usa para referirse a determinados lugares que han sufrido una sobreexplotación ambiental a costa de sacrificar sus recursos naturales y humanos, para lograr el aclamado desarrollo de proyectos de inversión sin control y sin regulación (González, 2017).

Inseguridad y dependencia alimentaria: es la falta de disponibilidad, de acceso, de estabilidad y de inocuidad de productos para el consumo, causada por la aplicación de políticas de libre mercado. Modificando la siembra, producción y consumo agrícola tradicional de los pueblos, haciendo que dependan de semillas e insumos de corporaciones, apropiación de los derechos de los pueblos por parte de grandes corporaciones (Jönsson, 2010). La dependencia alimentaria aumenta los monocultivos e impone el uso intensivo de insumos comprados y llevan al agricultor a someterse y competir con la agroindustria. Se observa que los alimentos, cada vez tienen origen extranjero o de grandes agroindustrias, disminuyendo la producción tradicional campesina. Lo que profundiza el hambre, la desnutrición, y la alimentación tóxica, debido a la proliferación de semillas transgénicas y la agricultura química promovidas por el gran capital agrícola. 
Movimientos sociales: se denominan como "el proceso de (re)constitución de una identidad colectiva, fuera del ámbito de la política institucional, por el cual se dota de sentido a la acción individual y colectiva" (Revilla, 1996, p. 2). El movimiento social es siempre la autorganización de los ciudadanos. Estos dejan ver una escasez en las identidades y caracteres colectivos preexistentes y un deseo de autoafirmación.

La modernidad/colonialidad: es la reflexión sobre las relaciones políticas, económicas, culturales $\mathrm{y}$ de donde se han formado las identidades de Latinoamérica. "la colonialidad constituye la superposición de los procesos económicos y políticos del capitalismo con los procesos de subjetivación y clasificación de los grupos sociales (lugar fundamental de la creación de lo blanco, lo negro y lo indígena)" (Espinosa, 2015). Espinosa cita a Quijano, para decir que "La colonialidad, ejerce un control de los distintos grupos sociales en torno a la idea de raza en su relación con las formas de trabajo (Quijano, 1999a). El proceso de la modernidad es constituido, por la articulación de las relaciones sociales a través de la idea de capital y de raza" (Espinosa, 2015, p. 111).

La identidad: es el proceso de perdida de propiedades y características de identidad étnico-racial, de economía en armonía con el ambiente, sus bases y modos comunitarios que se ven fracturados debido a los procesos de colonización de territorios ancestrales, para establecimiento de megaproyectos de monocultivos y extracción de minerales, facilitado por el capital de las transnacionales.

Papel de las mujeres: la ecología no reconoce ningún "rey de la bestias" ni ninguna creatura inferior, ya que estos conceptos vienen de nuestra mente. Pero, lo que si acepta es que los seres vivos son interdependientes y son complementarios, para perpetuar y estabilidad del orden natural, gradualmente estas ideas cambiaron, y con la división del trabajo tribal, comenzó a notarse la soberanía del varón sobre la mujer, para dominar (Bookchin, 1999).

\section{METODOLOGIA}

Es relevante estudiar estrategias latinoamericanas para el territorio en pensamiento y práctica, orientado en torno a los temas tierra, terreno y estado. Al hacerlo, su objetivo es contribuir al desmantelamiento y la reversión de las jerarquías coloniales que se basan en la imaginación y las prácticas territoriales dominantes (HALVORSEN, 2019).

La metodología a ser utilizada es la pesquisa cualitativa, por medio del método de estudio de caso, pues permite identificar el comportamiento de los individuos frente a un fenómeno, en un contexto social, las informaciones son obtenidas de documentos, registros de archivos de acuerdo con (Martínez, 2006, p.167). Comenzaremos con los conceptos y términos que son distinguidos en la Ecología Política Marxista y Decolonial. Este método es esencial para investigar problemas sociales. El tema a ser investigado fue seleccionado, como también una unidad de análisis y, en seguida, fueron recolectadas las informaciones teóricas y literarias, para generar el artículo final.

\section{RESULTADOS Y DISCUSIÓN}

Todos estos aspectos que se tomaron durante este recorrido conceptual, permitirán analizar la situación actual en la que se encuentra la población y el Lago de Tota, figura 1.

Figura 1: Generalidades del Lago de Tota. 


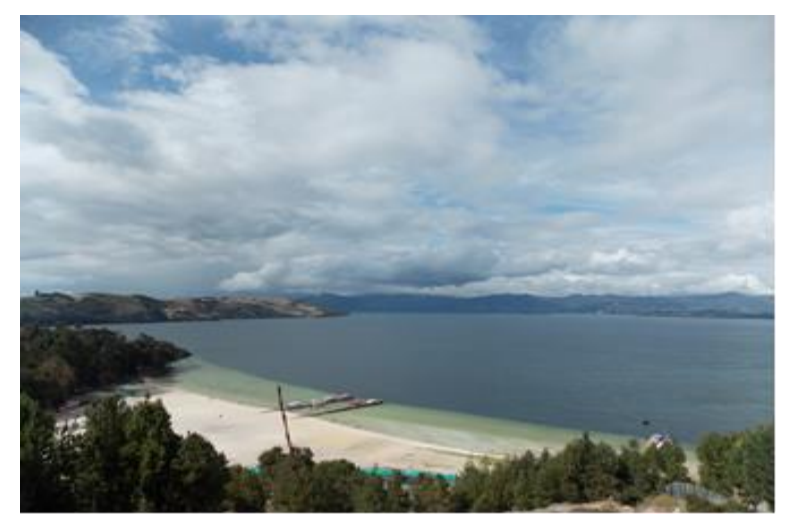

Fuente: Autora.

Sobre la región Andina colombiana se encuentra el departamento de Boyacá, dentro del cual está el Lago de Tota, cuenta con un área de $55 \mathrm{~km}^{2}$, está ubicado entre los municipios de Aquitania, Cuítiva y Tota, al oriente del departamento, en la Provincia de Sugamuxí, hace parte de un ecosistema de bosque alto andino, a 3.015 m.s.n.m. Nasce el río Upía. Es el cuerpo de agua de alta montaña más extenso e importante del país. Área de drenaje de $225.61 \mathrm{~km}^{2}$. Volumen de $1.920^{\prime} 000.000 \mathrm{~m}^{3}$ de agua. Longitud máxima de 12 kilómetros y su ancho de 6.5 kilómetros, y un perímetro de 47 kilómetros. Cuenta con 3 islas, las cuales son el complejo insular más grande del país para este tipo de ecosistema (Corpoboyacá, 2014). El espejo de agua tiene cerca de 6.000 hectáreas y profundidades máximas y media de 61 y 31.6 metros respectivamente. Se han identificado 6 especies de peces, 10 de herpetos, 23 de mamíferos y 83 especies de aves migratorias y endémicas. Tota expresa en lengua chibcha, labranza del rio, el Lago tiene 22.000 años (Aranguren, 2012, p.14).

Injusticia y racismo ambiental: en el Lago de Tota, la presencia del Estado, se evidencia en la ausencia para controlar los procesos de crecimiento de los asentamientos que vierten sus aguas residuales al Lago. Hasta ahora con los Planes de Ordenamiento Territorial, las administraciones locales han empezado a mostrar interés en el asunto y a definir los instrumentos para controlar todos los hechos inadecuados, que se vienen presentado desde hace varias décadas. Actualmente, la institucionalidad no se pronuncia de manera clara y directa, por ejemplo la empresa Acerías Paz del Río, propiedad de la siderúrgica brasileña Votorantim, desde 1952 ha tomado el agua para su proceso productivo, a través de la construcción de un túnel y hasta el 4 de junio de 2014, Corpoboyacá restringió en un $80 \%$ el uso de agua, como medida de protección. Así mismo, los acueductos municipales de Tota, Iza, Cuítiva, Aquitania, Sogamoso, Belencito, entre otros y Acerías Paz del Río, extraen aproximadamente "3.000 litros por segundo, sin brindar compensación para este humedal" (Wallace, 2012).

La dicotomía entre protección del medio ambiente y producción económica, que viven las comunidades campesinas del Lago, las lleva a dedicarse al monocultivo de cebolla, actividad económica que resulta más rentable. Además, la actitud de Corpoboyacá no ayuda mucho, ya que expresa que la responsabilidad de la situación ambiental que afecta al lago recae en los que allí residen, explotan los recursos ambientales y naturales (Corpoboyacá, 2005, p. 11). Sin embargo, es el Estado el que ha sido negligente para darle solución a la crisis y situación actual del recurso hídrico.

Conflictos y Problemáticas Ambientales: los modelos económicos, no respetan los ciclos normales del ecosistema natural, no consideran la sostenibilidad y equilibrio ambiental. El uso y manejo inadecuado del suelo, la utilización de fertilizantes en los cultivos, la despreocupación y abuso del sector turístico e industrial, incrementan los niveles de contaminación y ocasionan presión sobre el uso de los recursos disponibles (Corpoboyacá, 2004; Reyes et al., 2016).

Lo que actualmente vive la cuenca del Lago de Tota, es el resultado de las interacciones del tiempo, de las condiciones del mercado, de las características biofísicas y los aspectos culturales. 
Los problemas con la producción agropecuaria, se presenta por la falta de apropiación de pensamiento ambiental en la población rural.

Para Wallace (2012) en las riberas del Lago de Tota, se cultiva el 90\% de la cebolla larga que se consume en el país, ya que las condiciones climáticas de la zona son ideales y la abundancia de agua les garantiza a los cultivadores toda el agua que puedan necesitar. El uso de fungicidas (malatión, tebuconazol, difenoconazol y clorotalonilo) es complejo, se ha llegado a determinar en aguas superficiales que algunas muestras superaron el límite de cuantificación de la metodología (Mojica y Guerrero, 2013, p. 29). Además, una de las causas que complica aún más la situación del Lago, es la deforestación en las partes altas y la retención de aguas que se hace en algunos sectores por parte de las comunidades. Otra amenaza es la industria petrolera, ya que se han hecho exploraciones para encontrar petróleo.

Por otro lado, la oferta del sector turístico y por consiguiente de los servicios de hoteles, restaurantes gastronómicos, servicios pesqueros y náuticos, crea la tendencia a la concentración de estos en cercanías a los cascos urbanos y en áreas donde el suelo es utilizado para el desarrollo de la agricultura, generando incompatibilidad ambiental y estimulando de alguna forma los procesos de urbanización.

Desarrollo segregacionista: respecto a la situación del campesinado en Boyacá, "la cobertura de los servicios públicos de agua potable cubre solo un 22\%; en cuanto a la recolección de basuras la cobertura rural es de 15.7\%" (Gobierno del Departamento de Boyacá "Boyacá se atreve", 2012 2015, p. 76). Por este motivo llegan al Lago los vertimientos de aguas servidas producto de las actividades de pesca, ganadería, agricultura, hotelería y por el uso de la gallinaza en los cultivos. Se sabe que hay un documento CONPES (Consejo Nacional de Política Económica y Social) que confiere $\$ 40.940$ millones de pesos para la protección del Lago.

Por otra parte Corpoboyacá, sólo hasta el 24 de octubre de 2014, declara en Ordenación la Cuenca Hidrográfica del Lago de Tota (Sotelo, 2016). Existiendo un documento, desde el 2004, que proyecta mantener el equilibrio entre el aprovechamiento social, económico de los recursos y la conservación de la ordenación de la cuenca y concretamente del recurso hídrico.

Según, Sotelo en su tesis doctoral afirma que "se observa en las narrativas de los campesinos una fuerte oposición entre el progreso que tienen ahora y frente el no progreso que tenían hace más de 40 años, cuando no cultivaban cebolla" (Sotelo, 2016, p. 96). Por tal motivo se requiere de reorientar la identificación y propiedad ambiental, especialmente de los que viven y laboran en cercanías a las fuentes de agua, pues de ello depende la protección del recurso hídrico.

Zona de sacrificio: Franco, Delgado y Andrade, hacen referencia al Lago de Tota como vulnerable al fenómeno del calentamiento global. Así mismo en esté estudio afirman la desaparición de gran parte de la riqueza biológica, quedando la presencia escasa de mortiño y chite. Referente a fauna, deberían existir venados, armadillos, perros de monte, zorros, cusumbos, conejos, ardillas, comadrejas, musarañas, mariposas nacaradas, abejas, avispas, escarabajos, ranas, lagartijas, algunas serpientes y aves como colibríes, azulejos, baranqueros, pavas, torcazas, golondrinas, peces, entre otros. El principal uso que se le da a la fauna silvestre es de tipo artesanal, a excepción de la trucha que es la única que tiene fin comercial (Franco, Delgado y Andrade, 2013, p.74-75). Un estudio de la Pontificia Universidad Javeriana, encontró que en el Lago y sus drenajes, el pez graso (Rhizosomichthys totae), actualmente se considera extinta. La Red Mundial de Humedales, en el 2012, considero al Lago como uno de los de mayor afectación que tiene Colombia, producto de la acción del hombre, al punto de ser uno de los ecosistemas más amenazados del mundo (Wallace, 2012).

Inseguridad y dependencia alimentaria: antes de 1964, la superficie de tierra cultivable que bordea al Lago, se usaba para cultivar trigo, papa, cebada y se criaba ganado vacuno. Pero a partir de los años 1964 y 1966 se comienza el cultivo de cebolla en la región. Raymond explica que el cultivo de la cebolla, se produce por los bajos ingresos de los campesinos y por la presencia de 
plagas que atacaban el cultivo de papa. Así es que se intensifica el monocultivo de cebolla, por el clima frío, la existencia de suficiente agua, la fertilidad del suelo aledaño al Lago de Tota, la rentabilidad del cultivo, pues la obtención de cebolla se da entre tres y cuatro cosechas al año (Raymond, 1990).

Sin embargo, el aumento en los costos de la maquinaria, de los insumos agrícolas y las dinámicas del mercado han afectado al cultivo de cebolla y a los productores. La mayor rentabilidad la reciben los propietarios de grandes extensiones de tierra, quienes también realizan directamente el transporte terrestre del producto, a las ciudades principales. Mientras, los pequeños y medianos productores, sólo reciben lo que les pagan los intermediarios por su producto. La apertura económica indiscriminada y la sustitución de importaciones por parte del Estado, generaron más atraso en el campo.

Movimientos sociales: con respecto a este aspecto se encuentra que las acciones, para mitigar el impacto ambiental son escasas, solo un ciudadano de Aquitania logró con una acción de tutela prohibir radicalmente la extracción del agua del lago por parte de la empresa particular Acerías Paz del Río. Logrando que la Corte Constitucional mediante sentencia declarara inexequible el Decreto 1111 de 1952.Y concluyó que: “...todas las medidas contempladas en dicha norma plasman un modelo de gestión ambiental aplicado a un recurso natural estratégico, que se distancia abiertamente del manejo ambiental que conforme a la Constitución (Artículos. 8, 79 y 80) debe darse a los recursos naturales." (Sentencia C 094 de 2015).

La modernidad/colonialidad: las ideas que surgen en Europa se adoptan justamente para justificar proyectos propios. Los campesinos, se excusan en la necesidad de sobrevivir y en lo que la naturaleza les ha dado para su subsistencia y nadie se puede interponer ante esta lógica que habita en ellos. Otra consecuencia, es la progresiva desaparición de los campesinos, quienes históricamente han sembrado las tierras agrarias en el país, en muchas situaciones se quedan sin hacer lo que saben hacer (Tobasura, 2011, p. 653). La asistencia técnica productiva no está desarrollada y la existente sólo sirve a los propósitos de las empresas comercializadoras de insumos agrícolas.

La mayoría de las familias que viven alrededor del Lago, representa la mayor fuerza de trabajo, como afirma Raymond "la presencia de una economía netamente campesina que utiliza mano de obra familiar" (Raymond 1990), ha ayudado para que los productores consiguieran adaptarse rápidamente a las exigencias del mercado de la cebolla larga, como alternativa de producción en este lugar.

Hoy en día "el 1\% de las familias ricas en el campo concentran aproximadamente el $60 \%$ de la tierra apta para producir. Y al mismo tiempo ese poder económico de la tierra también da poder político", afirma Daniel Libreros (Agencia de Noticias de la Universidad Nacional de Colombia, 2014). Por lo tanto, los campesinos que poseen microparcelas, no logran la producción suficiente para generar los ingresos necesarios para su sustento, y se ven en la obligación de trabajar para los mayores productores de cebolla en la zona. Igualmente, en la región aledaña al Lago de Tota los procesos de modernidad, que se han dado es el uso desmedido de plaguicidas, fertilizantes y fungicidas, afectando el equilibrio ecológico y la salud pública (Raymond, 1990; Leroy et al., 2018).

La identidad: Fals Borda, en su libro 'El Hombre y la Tierra en Boyacá', manifiesta que una característica notoria de la población boyacense es la actitud pasiva que ha arraigado desde la época de la colonia, la cual tiene marcadas consecuencias políticas y económicas para la región en la actualidad, y explica que el liberalismo capitalista de mediados del siglo XIX, creo focos de minifundios, en los que se olvidaron las iniciativas de ayuda mutua entre trabajadores, provocando la alienación y el fraccionamiento del pueblo boyacense. Lo que le impide conformarse como fuerza que busque su propio cambio social, pues se ve afectado por procesos del minifundio, el aburguesamiento del campesinado, la manipulación y represión estatal (Fals, 1979; Amezquita, 2015). 
La irrupción de los españoles influenció la relación hombre-territorio, modificó profundamente, sus prácticas e ideologías. Se ha hablado y se han hecho intentos de reforma agraria, pero nunca se ha logrado que la tierra sea repartida equitativamente entre las poblaciones rurales. En el área del centro del país, es reconocido el movimiento de reindigenización muisca, en la época de la conquista eran cerca de 500 mil habitantes, que a la luz de estas nuevas maneras de constitución de ciudadanías, no fueron totalmente extinguidos, razón por la cual en la actualidad, protegidos por la Constitución e inspirados en los movimientos indígenas del siglo XXI, están restaurando su cultura ancestral.

Papel de las mujeres: según Díaz en Colombia "la participación de las mujeres rurales tiene una larga y variada trayectoria en el siglo XX y en los comienzos del XXI" (Díaz, 2002), período en el que las mujeres empiezan a participar en tiempos de luchas y movilizaciones en defensa de las comunidades (Pérez et al., 2012). Sin embargo el poder hegemónico siempre trata de invisibilizar los aportes y su papel que ha sido fundamental en muchos procesos sociales. En general, en el departamento de Boyacá las mujeres campesinas realizan los oficios artesanales con más frecuencia porque están más arraigadas a la preservación de la memoria y tienen un nexo más fuerte con la historia de los pueblos y grupos sociales que existían antes de la conquista hispana.

\section{CONSIDERACIONES FINALES}

De acuerdo a lo anterior y respondiendo a la preocupación de quienes son los que se ven perjudicados, con la situación actual del Lago de Tota, se encuentra que toda la población local se ve afectada, pues el Lago de Tota es un sistema que si alguna parte o componente del ecosistema falla, se va a ver reflejado en las otras partes que lo componen, por lo tanto es necesario generar alternativas frente a estas situaciones descritas, para dar a conocer otras formas de pensar y de interpretar el entorno, y poder generar la unión entre las comunidades que promuevan el progreso y desarrollo, frente al cuidado del medio ambiente, contrarrestando el desarrollo actual.

Para tener una administración desde lo local, existe debilidades, pues la población no tiene recursos económicos para pagar estudios que les sustente y demuestre un diagnóstico inicial y los posibles escenarios a los cuales se deben someter en casos extremos de contaminación y/o sequia del cuerpo de agua, aumento del turismo, ni cómo deben actuar para atender dichas situaciones.

No hay quien se responsabilicé e interese por conservar este importante ecosistema, o por generar espacios donde exista más control de los vertimientos, toma y extracción ilegal de agua, ampliación de la zona agrícola. En Colombia se requieren, políticas puntuales y participativas para atender la situación del sector rural y del campesino, ya que son en cierta forma el eje del desarrollo, pero no en las condiciones actuales en que se encuentran de olvido, pobreza y marginalización.

\section{REFERENCIAS}

AMEZQUITA, J. Departmental Institutions and Decentralised Cooperation in Boyacá. Perspectiva Geográfica, v.20, n.1, p.37-70, 2015

ARANGUREN, N. Memorias, foro «lago de tota claridad y soluciones sostenibles para su conservación», ceamder (U. Sto. Tomás, Tunja), Cuítiva, 21 de sep/2012 - Ver pág. 14, conferencia, Biólogo, Unidad de Ecología en Sistemas Acuáticos | UDESA, Universidad Pedagógica y Tecnológica de Colombia, U.P.T.C., Tunja Disponible en: http://www.ustatunja.edu.co/cestudios/files/MEMORIASTota-21 Septimebre.pdf. Consultado el 23 de Julio de 2018

BLAIKIE, P., BROOKFIELD, H. Land Degradation and Society, Methuen, London, 1987.

BOOKCHIN, M. La ecología de la Libertad. El surgimiento y la disolución de la jerarquía Nossa y Jara Editores. España. 1999 
CALDERÓN-CONTRERAS, R. Ecología política: hacia un mejor entendimiento de los problemas socioterritoriales. Economía, sociedad y territorio, v. 13, n. 42, p. 561-569, 2013.

DANE-Departamento Administrativo Nacional de Estadística. Primer censo del cultivo de cebolla larga. Ministerio de Agricultura y Desarrollo Rural. 2001.

DÍAZ, D. Situación de la mujer rural colombiana: perspectiva de género. Cuadernos Tierra y Justicia, n.9. Bogotá: ILSA. 2002.

ESCOBAR, A. Territorios de diferencia: lugar, movimiento, vida y redes. Universidad de Carolina del Norte, Chapel Hill. 2010.

ESPINOSA, S. Identidad y otredad en la teoría descolonial de Aníbal Quijano. Ciencia Política. Revista Ciencia Política, v. 10, n.20, p. 107-130, 2015

FONTAINE, G. Enfoques Conceptuales y metodológicos para una sociología de los conflictos ambientales, en: Cardenas M. y Rodriguez M. (eds), Guerra, Sociedad y Medio Ambiente. Foro Nacional Ambiental. Bogotá, 2004. Disponible en: https://library.fes.de/pdf-files/bueros/kolumbien/01993/12.pdf. Consultado el 1 de Agosto de 2018

GOBIERNO DEPARTAMENTAL. Plan de Gobierno del Departamento de Boyacá "Boyacá se atreve”, 2012 - 2015. Gobernación de Boyacá.

GONZÁLEZ, I. Crímenes contra la humanidad en el cómic periodístico de Joe Sacco: el caso de días de destrucción, días de revuelta. Universidad de León. 2017

HALVORSEN, S. Decolonising territory: Dialogues with Latin American knowledges and grassroots strategies. Progress in Human Geography, v. 43, n. 5, p. 790-814, 2019.

HARDIN, G. The tragedy of the commons. Source: Science, New Series. v.162, n.3859, p. 1243-1248,1968.

JÖNSSON, M. Desafíos de la globalización los sistemas agroalimentarios en América Latina. Ministry of Foreign Affairs of Denmark, DANIDA, Bolivia, 2010.

LAO-MONTES, A. Crisis de la civilización occidental capitalista y movimientos. Universidad de Massachusetts.

LEFF, E. La ecología política en América Latina. Un campo en construcción. Polis. Revista Latinoamericana, n. 5, 2003.

LEROY, D., MALAXECHEBARÍA, A. M. G., ANTOINE, J. M., \& ANGÉLIAUME-DESCAMPS, A. Gouvernance territoriale de l'environnement et conflits d'usage. Le cas du bassin versant du lac de Tota (Boyacá, Andes colombiennes). EchoGéo, v.43, 2018.

MARTÍNEZ, P. El método de estudio de caso Estrategia metodológica de la investigación científica. Revista científica Pensamiento y Gestión, v.20, 2011.

MASSA-SÁNCHEZ, P., CISNE ARCOS, R. D., MALDONADO, D. Minería a gran escala y conflictos sociales: un análisis para el sur de Ecuador. Problemas del desarrollo, v.49, n.194, p.119-141, 2018.

MOJICA, A.; GUERRERO, J. Evaluación del movimiento de plaguicidas hacia la cuenca del Lago de Tota, Colombia. Revista Colombiana de Química, v. 42, n. 2, p. 1-27, 2013.

PACHECO, T. Desigualdad, injusticia ambiental y racismo: una lucha que transciende el color de la piel. Polis: Revista Latinoamericana, v.16, p.18, 2007.

PÉREZ, A., DOMíngueZ, S., GALleGO, M. M. C. Políticas públicas para la mujer en Colombia: La doble condición de madre y trabajadora en la legislación del siglo XX. Entramado, v.8, n.1, p.72-88, 2012

RAYMOND, P. El Lago de Tota ahogado en cebolla. Estudio Socioeconómico de la Cuenca cebollera del Lago de Tota. Pontificia Universidad Javeriana: Bogotá. 1990.

REVILLA, M. El concepto de movimiento social: Acción, identidad y sentido. Última Década, n.5, Centro de Estudios Sociales Valparaíso, Chile. 1996.

REYES, Y., VERGARA, I., TORRES, O., LAGOS, M. D., JIMENEZ, E. E. G. Contaminación por metales pesados: Implicaciones en salud, ambiente y seguridad alimentaria. Ingeniería Investigación y Desarrollo: I2+ D, v.16, n.2, p.6677, 2016.

SOTELO, D. Las comunidades rurales y su significado de progreso. Casos del lago de la cocha y el lago de tota. Colombia. 2016. Disponible en: https://repositorio.uptc.edu.co/bitstream/001/1565/1/TGT-301.pdf. Consultado el 1 de Agosto de 2018

STRACCIA, P. H. Ecología política: aportes de la sociología y de la antropología. Cuadernos de Desarrollo Rural, v. 16, n. 84, 2019. 
TOBASURA, I. De campesinos a empresarios: la retórica neoliberal de la política agraria en Colombia. Espacio Abierto, v.20, n.4, p.641-647, 2011. Disponible en: http://www.redalyc.org/pdf/122/12220531005.pdf. Consultado el 3 de Agosto de 2018

TORRES, F. Seguridad alimentaria: una explicación sobre prevalencia de los desequilibrios en México. Secretaria de desarrollo agrario, territorial y urbano México. 2014.

UNIVERSIDAD NACIONAL DE COLOMBIA. Agencia de Noticias. Concentración de tierra, el mayor problema agrario en Colombia, El Espectador. 2014.

VALCÁRCEL, M. Génesis y evolución del concepto y enfoques sobre el desarrollo. Pontificia Universidad Católica del Perú. 2006.

WALLACE, A. El Lago de Tota, el más contaminado del mundo, ahora amenazado por el petróleo, BBC, Londres, ago. 2012.

WALTER, M. Conflictos ambientales, socioambientales, ecológico distributivos, de contenido ambiental... Reflexionando sobre enfoques y definiciones. Universitat Autònoma de Barcelona. 2009.

The author stated that had: a) actively participated in the discussion of the results; and b) Review and approval of the final version of the paper.

This work is licensed under a Creative Commons Attribution 4.0 International License. CC-BY 